\title{
A Study of a Certain Subclass of Hurwitz-Lerch-Zeta Function Related to a Linear Operator
}

\author{
F. Ghanim \\ Department of Mathematics, College of Sciences, University of Sharjah, Sharjah, UAE \\ Correspondence should be addressed to F. Ghanim; firas.zangnaa@gmail.com
}

Received 8 July 2013; Accepted 23 August 2013

Academic Editor: Mohamed Amal Aouf

Copyright (C) 2013 F. Ghanim. This is an open access article distributed under the Creative Commons Attribution License, which permits unrestricted use, distribution, and reproduction in any medium, provided the original work is properly cited.

By using a linear operator with Hurwitz-Lerch-Zeta function, which is defined here by means of the Hadamard product (or convolution), the author investigates interesting properties of certain subclasses of meromorphically univalent functions in the punctured unit $\operatorname{disk} U^{*}$.

\section{Introduction}

A meromorphic function is a single-valued function that is analytic in all but possibly a discrete subset of its domain, and at those singularities, it must go to infinity like a polynomial (i.e., these exceptional points must be poles and not essential singularities). A simpler definition states that a meromorphic function $f(z)$ is a function of the form

$$
f(z)=\frac{g(z)}{h(z)}
$$

where $g(z)$ and $h(z)$ are entire functions with $h(z) \neq 0$ (see [1, page 64]). A meromorphic function therefore may only have finite-order, isolated poles and zeros and no essential singularities in its domain. A meromorphic function with an infinite number of poles is exemplified by $\csc (1 / z)$ on the punctured disk $U^{*}=\{z: 0<|z|<1\}$. An equivalent definition of a meromorphic function is a complex analytic map to the Riemann sphere. Another definition for a meromorphic function is the following (see [2]).

Definition 1. A function $f$ on an open set $\Omega$ is meromorphic if there exists a discrete set of points $S=\{z: z \in \Omega\}$ such that $f$ is holomorphic on $\Omega-S$ and has poles at each $z \in S$. Furthermore, $f$ is meromorphic in the extended complex plane if $F(z)=f(1 / z)$ is either meromorphic or holomorphic at 0 . In this case, we say that $f$ has a pole or is holomorphic at infinity.
Example 2. The Gamma function is meromorphic in the whole complex plane.

Example 3. All rational functions such as

$$
f(z)=\frac{z^{3}-2 z+10}{z^{5}+3 z-1}
$$

are meromorphic on the whole complex plane.

Example 4. The functions $f_{1}(z)=e^{z} / z$ and $f_{2}(z)=\sin z /$ $(1-z)^{2}$ are meromorphic on the whole complex plane.

Example 5. The function $f(z)=e^{1 / z}$ is defined in the whole complex plane except for the origin 0 . However, 0 is not a pole of this function, rather an essential singularity. Thus, this function is not meromorphic in the whole complex plane.

Example 6. The complex logarithm function $f(z)=\ln z$ is not meromorphic on the whole complex plane, as it cannot be defined on the whole complex plane except for an isolated set of points.

The aim of this paper is to investigate interesting properties of certain subclasses of meromorphically univalent functions with linear operator which are defined here by means of the Hadamard product (or convolution) in the punctured unit disk $U^{*}$. 


\section{Preliminaries}

Let $\Sigma$ denote the class of meromorphic functions $f(z)$ normalized by

$$
f(z)=\frac{1}{z}+\sum_{n=1}^{\infty} a_{n} z^{n}
$$

which are analytic in the punctured unit disk $U^{*}$. For $0 \leq \beta$, we denote by $S^{*}(\beta)$ and $k(\beta)$ the subclasses of $\Sigma$ consisting of all meromorphic functions which are, respectively, starlike of order $\beta$ and convex of order $\beta$ in $U^{*}$.

For functions $f_{j}(z)(j=1 ; 2)$ defined by

$$
f_{j}(z)=\frac{1}{z}+\sum_{n=1}^{\infty} a_{n, j} z^{n}
$$

we denote the Hadamard product (or convolution) of $f_{1}(z)$ and $f_{2}(z)$ by

$$
\left(f_{1} * f_{2}\right)=\frac{1}{z}+\sum_{n=1}^{\infty} a_{n, 1} a_{n, 2} z^{n}
$$

Let us define the function $\widetilde{\phi}(\alpha, \beta ; z)$ by

$$
\widetilde{\phi}(\alpha, \beta ; z)=\frac{1}{z}+\sum_{n=0}^{\infty} \frac{(\alpha)_{n+1}}{(\beta)_{n+1}} z^{n},
$$

for $\beta \neq 0,-1,-2, \ldots$, and $\alpha \in \mathbb{C} \backslash\{0\}$, where $(\lambda) n=\lambda(\lambda+1)_{n+1}$ is the Pochhammer symbol. We note that

$$
\widetilde{\phi}(\alpha, \beta ; z)=\frac{1}{z^{2}} F_{1}(1, \alpha, \beta ; z),
$$

where

$$
{ }_{2} F_{1}(b, \alpha, \beta ; z)=\sum_{n=0}^{\infty} \frac{(b)_{n}(\alpha)_{n}}{(\beta)_{n}} \frac{z^{n}}{n !}
$$

is the well-known Gaussian hypergeometric function.

We recall here a general Hurwitz-Lerch-Zeta function, which is defined in $[3,4]$ by the following series:

$$
\Phi(z, t, a)=\frac{1}{a^{t}}+\sum_{n=1}^{\infty} \frac{z^{n}}{(n+a)^{t}}
$$

$\left(a \in \mathbb{C} \backslash \mathbb{Z}_{0}^{-}, \mathbb{Z}_{0}^{-}=\{0,-1,-2, \ldots\} ; t \in \mathbb{C}\right.$ when $z \in U=U^{*} \subset$ $\{0\} ; \mathfrak{R}(t)>1$ when $z \in \partial U)$.

Important special cases of the function $\Phi(z, t, a)$ include, for example, the Riemann Zeta function $\zeta(t)=\Phi(1, t, 1)$, the Hurwitz Zeta function $\zeta(t, a)=\Phi(1, t, a)$, the Lerch Zeta function $l_{t}(\zeta)=\Phi\left(\exp ^{2 \pi i \xi}, t, 1\right),(\xi \in \mathbb{R}, \mathfrak{R}(t)>1)$, and the polylogarithm $L_{t}^{i}(z)=z \Phi(z, t, a)$. Recent results on $\Phi(z, t, a)$ can be found in the expositions $[5,6]$. By making use of the following normalized function we define

$$
\begin{aligned}
G_{t, a}(z) & =(1+a)^{t}\left[\Phi(z, t, a)-a^{t}+\frac{1}{z(1+a)^{t}}\right] \\
& =\frac{1}{z}+\sum_{n=1}^{\infty}\left(\frac{1+a}{n+a}\right)^{t} z^{n}
\end{aligned}
$$

$\left(z \in U^{*}\right)$.
Corresponding to the functions $G_{t, a}(z)$ and using the Hadamard product for $f(z) \in \Sigma$, we define a new linear operator $L_{t, a}(\alpha, \beta)$ on $\Sigma$ by the following series:

$$
\begin{aligned}
L_{a}^{t}(\alpha, \beta) f(z) & =\phi(\alpha, \beta ; z) * G_{t, a}(z) \\
& =\frac{1}{z}+\sum_{n=1}^{\infty} \frac{(\alpha)_{n+1}}{(\beta)_{n+1}}\left(\frac{1+a}{n+a}\right)^{t} a_{n} z^{n}
\end{aligned}
$$

$\left(z \in U^{*}\right)$.

The meromorphic functions with the generalized hypergeometric functions were considered recently by many others; see, for example, [7-12].

It follows from (11) that

$$
\begin{aligned}
z\left(L_{a}^{t}(\alpha, \beta) f(z)\right)^{\prime}= & \alpha\left(L_{a}^{t}(\alpha+1, \beta) f(z)\right) \\
& -(\alpha+1) L_{a}^{t}(\alpha, \beta) f(z) .
\end{aligned}
$$

We denote by the class $\Sigma_{\alpha, \beta}^{a, t}(\gamma, \delta, \mu, \lambda)$ of all functions $f(z) \in \Sigma$ such that

$$
\begin{aligned}
& \mathfrak{R}\left\{(1-\lambda)\left(\frac{L_{a}^{t}(\alpha, \beta) f(z)}{L_{a}^{t}(\alpha, \beta) g(z)}\right)^{\mu}\right. \\
& \left.\quad+\lambda\left(\frac{L_{a}^{t}(\alpha+1, \beta) f(z)}{L_{a}^{t}(\alpha+1, \beta) g(z)}\right) \cdot\left(\frac{L_{a}^{t}(\alpha, \beta) f(z)}{L_{a}^{t}(\alpha, \beta) g(z)}\right)^{\mu-1}\right\}>\gamma,
\end{aligned}
$$

where $g(z) \in \sum$ satisfies the following condition:

$$
\Re\left\{\frac{L_{a}^{t}(\alpha, \beta) g(z)}{L_{a}^{t}(\alpha+1, \beta) g(z)}\right\}>\delta \quad\left(0 \leq \delta<1, z \in U^{*}\right),
$$

where $\gamma$ and $\mu$ are real numbers such that $0 \leq \gamma<1, \mu>0$, and $\lambda \in \mathbb{C}$ with $\mathfrak{R}\{\lambda\}>0$.

To establish our main results, we need the following lemmas.

Lemma 7 (see [13]). Let $\Omega$ be a set in the complex plane $\mathbb{C}$ and let the function $\Psi: \mathbb{C}^{2} \rightarrow \mathbb{C}$ satisfy the condition $\Psi\left(i r_{2}, s_{1}\right) \notin$ $\Omega$ for all real $r_{2}, s_{1} \leq\left(1+r_{2}^{2}\right) / 2$. If $q(z)$ is analytic in $U^{*}$ with $q(0)=1$ and $\Psi\left(q(z), z q^{\prime}(z)\right) \in \Omega, z \in U^{*}$, then $\mathfrak{R}\{q(z)\}>0$.

Lemma 8 (see [14]). If $q(z)$ is analytic in $U^{*}$ with $q(0)=1$, and if $\lambda \in \mathbb{C} \backslash\{0\}$ with $\mathfrak{R}\{\lambda\}>0$, then $\mathfrak{R}\left\{q(z)+\lambda z q^{\prime}(z)\right\}>\gamma$, $(0 \leq \gamma<1)$ implies $\mathfrak{R}\{q(z)\}>\gamma+(1-\gamma)(2 \varepsilon-1)$, where $\varepsilon$ is given by

$$
\varepsilon=\varepsilon(\Re \lambda)=\int_{0}^{1}\left(1+t^{\Re \lambda}\right)^{-1} d t
$$

which is increasing function of $\Re\{\lambda\}$ and $1 / 2 \leq \varepsilon<1$. The estimate is sharp in the sense that the bound cannot be improved.

For real or complex numbers $a, b, c,\left(c \notin z_{0}^{-}\right)$, the Gauss hypergeometric function is defined by

$$
\begin{aligned}
& { }_{2} F_{1}(a, b, c ; z) \\
& \quad=1+\frac{a b}{c} \frac{z}{1 !}+\frac{a(a+1) b(b+1)}{c(c+1)} \frac{z^{2}}{2 !}+\cdots .
\end{aligned}
$$


One notes that the above series converges absolutely for $z \in U^{*}$ and hence represents an analytic function in the unit disc $U^{*}$ (see, for details, [15, Chapter 14]).

Each of the identities (asserted by Lemma 9) is fairly well known (cf., e.g., [15, Chapter 14]).

Lemma 9. For real or complex parameters $a, b, c,\left(c \notin z_{0}^{-}\right)$, then

$$
\begin{aligned}
\int_{0}^{1} t^{b-1} & (1-t)^{c-b-1}(1-t z)^{-a} d t \\
= & \frac{\Gamma(b) \Gamma(c-b)}{\Gamma(c)}{ }_{2} F_{1}(a, b, c ; z)
\end{aligned}
$$

$(\mathfrak{R}(c)>\Re(b)>0)$,

$$
\begin{aligned}
&{ }_{2} F_{1}(a, b, c ; z)=(1-z){ }_{2}^{-a} F_{1}\left(a, c-b, c ; \frac{z}{z-1}\right), \\
&{ }_{2} F_{1}(a, b, c ; z)={ }_{2} F_{1}(b, c, c ; z), \\
&{ }_{2} F_{1}\left(1,1,2 ; \frac{1}{2}\right)=2 \ln 2 .
\end{aligned}
$$

\section{Main Results}

Theorem 10. Let $f(z) \in \Sigma_{\alpha, \beta}^{a, t}(\gamma, \delta, \mu, \lambda), \alpha \in \mathbb{R} \backslash\{0\}$ and $\lambda \geq 0$. Then

$$
\mathfrak{R}\left(\frac{L_{a}^{t}(\alpha, \beta) g(z)}{L_{a}^{t}(\alpha, \beta) g(z)}\right) \geq \frac{2 \alpha \gamma \mu+\delta \lambda}{2 \alpha \mu+\delta \lambda}
$$

$\left(0 \leq \gamma<1, \mu>0, z \in U^{*}\right)$, where the function $g(z) \in \Sigma$ satisfies condition (14).

Proof. Let $\xi=(2 \alpha \gamma \mu+\delta \lambda) /(2 \alpha \mu+\delta \lambda)$, and we define the function $q(z)$ by

$$
q(z)=\frac{1}{(1-\xi)}\left\{\left(\frac{L_{a}^{t}(\alpha, \beta) f(z)}{L_{a}^{t}(\alpha, \beta) g(z)}\right)^{\mu}-\xi\right\} .
$$

Then $q(z)$ is analytic in $U^{*}$ and $q(0)=1$. If we set

$$
h(z)=\frac{L_{a}^{t}(\alpha, \beta) g(z)}{L_{a}^{t}(\alpha+1, \beta) g(z)},
$$

then by the hypothesis $\Re\{h(z)\}>\delta$. Differentiating (19) and using the identity (12), we have

$$
\begin{aligned}
(1-\lambda) & \left(\frac{L_{a}^{t}(\alpha, \beta) f(z)}{L_{a}^{t}(\alpha, \beta) g(z)}\right)^{\mu} \\
& +\lambda \frac{L_{a}^{t}(\alpha+1, \beta) f(z)}{L_{a}^{t}(\alpha+1, \beta) g(z)}\left(\frac{L_{a}^{t}(\alpha, \beta) f(z)}{L_{a}^{t}(\alpha, \beta) g(z)}\right)^{\mu-1} \\
= & {[\xi+(1-\xi) q(z)]+\frac{\lambda(1-\xi)}{\alpha \mu} h(z) z q^{\prime}(z) }
\end{aligned}
$$

Let us define the function $\Psi(r, s)$ by

$$
\Psi(r, s)=\xi+(1-\xi) r+\frac{\lambda(1-\xi)}{\alpha \mu} h(z) s .
$$

Using (21) and the fact that $f(z) \in \sum_{\alpha, \beta}^{a, t}(\gamma, \delta, \mu, \lambda)$, we obtain

$$
\begin{gathered}
\left\{\Psi\left(q(z), z q^{\prime}(z)\right) ; z \in U^{*}\right\} \subset \Omega \\
=\{w \in C: \Re(w)>\gamma\} .
\end{gathered}
$$

Now for all real $r_{2}, s_{1} \leq\left(1+r_{2}^{2}\right) / 2$, we have

$$
\begin{aligned}
\mathfrak{R}\left\{\Psi\left(i r_{2}, s_{1}\right)\right\} & =\xi+\frac{\lambda(1-\xi)}{\alpha \mu} \mathfrak{R}\{h(z)\} \\
& \leq \xi-\frac{\lambda \delta(1-\xi)\left(1+r_{2}^{2}\right)}{2 \alpha \mu} \\
& \leq \xi-\frac{\lambda \delta(1-\xi)}{2 \alpha \mu}=\gamma .
\end{aligned}
$$

Hence for each $z \in U^{*}, \Psi\left(i r_{2}, s_{1}\right) \notin \Omega$. Thus by Lemma 7, we have $\mathfrak{R}\{q(z)\}>0$, and hence

$$
\Re\left(\frac{L_{a}^{t}(\alpha, \beta) f(z)}{L_{a}^{t}(\alpha, \beta) g(z)}\right)^{\mu}>\xi, \quad z \in U^{*} .
$$

This proves Theorem 10 .

Corollary 11. Let the functions $f(z)$ and $g(z)$ be in $\Sigma$, and let $g(z)$ satisfy condition (14). If $\alpha \in \mathbb{R} \backslash\{0\}, \lambda \geq 0$, and

$$
\mathfrak{R}\left\{(1-\lambda) \frac{L_{a}^{t}(\alpha, \beta) f(z)}{L_{a}^{t}(\alpha, \beta) g(z)}+\lambda \frac{L_{a}^{t}(\alpha+1, \beta) f(z)}{L_{a}^{t}(\alpha+1, \beta) g(z)}\right\}>\gamma,
$$

$0 \leq \gamma<1, z \in U^{*}$, then

$$
\mathfrak{R}\left\{\frac{L_{a}^{t}(\alpha+1, \beta) f(z)}{L_{a}^{t}(\alpha+1, \beta) g(z)}\right\}>\xi=\frac{\gamma(2 \alpha+\delta)+\delta(\lambda-1)}{2 \alpha+\lambda \delta} .
$$

Proof. We have

$$
\begin{aligned}
& \lambda \frac{L_{a}^{t}(\alpha+1, \beta) f(z)}{L_{a}^{t}(\alpha+1, \beta) g(z)} \\
& =\left[(1-\lambda) \frac{L_{a}^{t}(\alpha, \beta) f(z)}{L_{a}^{t}(\alpha, \beta) g(z)}+\lambda \frac{L_{a}^{t}(\alpha+1, \beta) f(z)}{L_{a}^{t}(\alpha+1, \beta) g(z)}\right] \\
& +(\lambda-1) \frac{L_{a}^{t}(\alpha, \beta) f(z)}{L_{a}^{t}(\alpha, \beta) g(z)} .
\end{aligned}
$$

Since $\lambda>1$ making use of (27) and (19) (for $\mu=1$ ), we deduce that

$$
\mathfrak{R}\left\{\frac{L_{a}^{t}(\alpha+1, \beta) f(z)}{L_{a}^{t}(\alpha+1, \beta) g(z)}\right\}>\xi=\frac{\gamma(2 \alpha+\delta)+\delta(\lambda-1)}{2 \alpha+\lambda \delta} .
$$


Corollary 12. Let $\lambda \in \mathbb{C} \backslash\{0\}$ with $\Re\{\lambda\}>0$ and $\alpha \in \mathbb{R} \backslash\{0\}$. implies If $f(z) \in \sum$ satisfies the following condition:

$$
\begin{aligned}
& \Re(1-\lambda)\left(z L_{a}^{t}(\alpha, \beta) f(z)\right)^{\mu}+\lambda\left(z L_{a}^{t}(\alpha+1, \beta) f(z)\right) \\
&\left.\cdot\left(z L_{a}^{t}(\alpha, \beta) f(z)\right)^{\mu-1}\right\}>\gamma
\end{aligned}
$$

$\left(0 \leq \gamma<1, \mu>0, z \in U^{*}\right)$, then

$$
\mathfrak{R}\left\{z L_{a}^{t}(\alpha, \beta) f(z)\right\}^{\mu}>\frac{2 \mu \alpha \gamma+\Re\{\lambda\}}{2 \mu \alpha+\mathfrak{R}\{\lambda\}} .
$$

Further, if $\lambda \geq 1, \alpha \in \mathbb{R} \backslash\{0\}$, and $f(z) \in \sum$ satisfies

$$
\mathfrak{R}\left\{(1-\lambda) z L_{a}^{t}(\alpha, \beta) f(z)+\lambda\left(z L_{a}^{t}(\alpha+1, \beta) f(z)\right)\right\}>\gamma,
$$

then

$$
\begin{aligned}
\Re\left\{z L_{a}^{t}(\alpha+1, \beta) f(z)\right\} & >\frac{(2 a+1) \gamma+\lambda-1}{2 a+\lambda} \\
(0 & \left.\leq \gamma<1, z \in U^{*}\right) .
\end{aligned}
$$

Proof. The results (19) and (20) follow by putting $g(z)=1 / z$ in Theorem 10 and Corollary 11, respectively.

Remark 13. (i) Putting $\lambda=1$ and $\alpha, \beta>0$ in Corollary 12, we have that

$$
\mathfrak{R}\left\{z L_{a}^{t}(\alpha+1, \beta) f(z) \cdot\left(z L_{a}^{t}(\alpha, \beta) f(z)\right)^{\mu-1}\right\}>\gamma
$$

implies

$$
\mathfrak{R}\left\{z L_{a}^{t}(\alpha, \beta) f(z)\right\}^{\mu}>\frac{2 \alpha \gamma+\mathfrak{R}\{\lambda\}}{2 \alpha+\mathfrak{R}\{\lambda\}}, \quad z \in U^{*} .
$$

(ii) For $\lambda \in \mathbb{C} \backslash\{0\}$ with $\Re\{\lambda\}>0, \mu=1$, and $\alpha, \beta>0$ in Corollary 12 , we have that

$$
\begin{aligned}
\Re & \left\{(1-\lambda) z L_{a}^{t}(\alpha, \beta) f(z)\right. \\
& \left.+\lambda\left(z L_{a}^{t}(\alpha+1, \beta) f(z)\right)\right\}>\gamma
\end{aligned}
$$

$(0 \leq \gamma<1)$ implies

$$
\mathfrak{R}\left\{z L_{a}^{t}(\alpha, \beta) f(z)\right\}>\frac{2 \alpha \gamma+\mathfrak{R}\{\lambda\}}{2 \alpha+\mathfrak{R}\{\lambda\}}, \quad z \in U^{*} .
$$

Choosing $t=0$ and $\alpha=\beta=1$ appropriately in Corollary 12, we obtain the following results.

(iii) For $\lambda=1, t=0$, and $\alpha=\beta=1$ in Corollary 12, we have that

$$
\begin{array}{r}
\Re\left\{\frac{z f^{\prime}(z)}{f(z)}(z f(z))^{\mu}\right\}>\gamma, \\
(0 \leq \gamma<1, \mu>0)
\end{array}
$$

$$
\Re\{z f(z)\}^{\mu}>\frac{2 \gamma \mu+1}{2 \mu+1}, \quad z \in U^{*} .
$$

(iv) For $\lambda \in \mathbb{C} \backslash\{0\}$ with $\mathfrak{R}\{\lambda\}>0, \mu=1, t=0$, and $\alpha=\beta=1$ in Corollary 12, we have that

$$
\mathfrak{R}\left\{(1-\lambda) z f(z)+\lambda\left(z^{2} f^{\prime}(z)\right)\right\}>\gamma
$$

$(0 \leq \gamma<1, \mu>0)$ implies

$$
\Re\{z f(z)\}>\frac{2 \gamma+\mathfrak{R}\{\lambda\}}{2+\mathfrak{R}\{\lambda\}}, \quad z \in U^{*} .
$$

(v) Replacing $f(z)$ by $-z f^{\prime}(z)$ in the result (ii), we have that

$$
-\Re\left\{(1-\lambda) z^{2} f(z)+\lambda\left(z^{3} f^{\prime \prime}(z)\right)\right\}>\gamma
$$

implies

$$
-\Re\left\{z^{2} f^{\prime}(z)\right\}>\frac{2 \gamma+\mathfrak{R}\{\lambda\}}{2+\mathfrak{R}\{\lambda\}}, \quad z \in U^{*} .
$$

(vi) For $\lambda \in \mathbb{R}$ with $\lambda \geq 1, \mu=1, t=0$, and $\alpha=\beta=1$ in Corollary 12 , we have that

$$
\mathfrak{R}\left\{(1-\lambda) z f(z)+\lambda\left(z^{2} f^{\prime}(z)\right)\right\}>\gamma
$$

implies

$$
\Re\{z f(z)\}>\frac{3 \gamma+\lambda-1}{2+\lambda} .
$$

Theorem 14. Let $\lambda \in \mathbb{C} \backslash\{0\}$ with $\Re\{\lambda\}>0$ and $\alpha \in \mathbb{R} \backslash\{0\}$. If $f(z) \in \Sigma$ satisfies the following condition:

$$
\begin{aligned}
& \mathfrak{R}\left\{(1-\lambda)\left(z L_{a}^{t}(\alpha, \beta) f(z)\right)^{\mu}\right. \\
&\left.+\lambda\left(z L_{a}^{t}(\alpha+1, \beta) f(z)\right) \cdot\left(z L_{a}^{t}(\alpha, \beta) f(z)\right)^{\mu-1}\right\}>\gamma \\
&\left(0 \leq \gamma<1, \mu>0, z \in U^{*}\right),
\end{aligned}
$$

then

$$
\mathfrak{R}\left\{z L_{a}^{t}(\alpha, \beta) f(z)\right\}^{\mu}>\gamma+(1-\gamma)(2 \rho-1),
$$

where

$$
\rho=\frac{1}{2}{ }_{2} F_{1}\left(1,1, \frac{\mu a}{\Re\{\lambda\}}, \frac{1}{2}\right), \quad z \in U^{*} .
$$

Proof. Let

$$
q(z)=\left(z L_{a}^{t}(\alpha, \beta) f(z)\right)^{\mu} .
$$


Then $q(z)$ is analytic in $U^{*}$ with $q(0)=1$. Differentiating $q(z)$ with respect to $z$ and using the identity (12), we obtain

$$
\begin{aligned}
& (1-\lambda)\left(z L_{a}^{t}(\alpha, \beta) f(z)\right)^{\mu}+\lambda\left(z L_{a}^{t}(\alpha+1, \beta) f(z)\right) \\
& \cdot\left(z L_{a}^{t}(\alpha, \beta) f(z)\right)^{\mu-1} \\
& =q(z)+\frac{\lambda z q^{\prime}(z)}{\mu a}
\end{aligned}
$$

so that by the hypothesis (47), we have

$$
\mathfrak{R}\left\{q(z)+\frac{\lambda z q^{\prime}(z)}{\mu a}\right\}>\gamma .
$$

In view Lemma 8 , this implies that

$$
\mathfrak{R}\{q(z)\}>\gamma+(1-\gamma)(2 \rho-1),
$$

where

$$
\rho=\rho(\Re\{\lambda\})=\int_{0}^{1}\left(1+t^{\Re\{\lambda\} / \mu a}\right)^{-1} d t .
$$

Putting $\mathfrak{R}\{\lambda\}=\lambda_{1}>0$, we have

$$
\rho=\int_{0}^{1}\left(1+t^{\Re\{\lambda\} / \mu \alpha}\right)^{-1} d t=\frac{\mu \alpha}{\lambda_{1}} \int_{0}^{1} u^{\left(\mu \alpha / \lambda_{1}\right)-1}(1+u)^{-1} d u .
$$

Using (17)-(18), we obtain

$$
\rho={ }_{2} F_{1}\left(1, \frac{\mu \alpha}{\lambda_{1}} ; \frac{\mu \alpha}{\lambda_{1}}+1 ;-1\right) \rho^{*}=\frac{1}{2}{ }_{2} F_{1}\left(1,1, \frac{\alpha}{\lambda}+1, \frac{1}{2}\right) .
$$

Thus the proof of Theorem 14 is complete.

Corollary 15. Let $\lambda \in \mathbb{R}, \mu=1$ with $\lambda>1$. If $f(z) \in \Sigma$ satisfies

$$
\begin{aligned}
\Re & (1-\lambda)\left(z L_{a}^{t}(\alpha, \beta) f(z)\right) \\
& \left.+\lambda\left(z L_{a}^{t}(\alpha+1, \beta) f(z)\right)\right\}>\gamma
\end{aligned}
$$

$\left(0 \leq \gamma<1, \alpha \in \mathbb{R} \backslash\{0\}, z \in U^{*}\right)$, then

$$
\begin{aligned}
& \mathfrak{R}\left\{\left(z L_{a}^{t}(\alpha+1, \beta) f(z)\right)\right\} \\
& >\gamma+(1-\gamma)\left(2 \rho^{*}-1\right)\left(1-\lambda^{-1}\right),
\end{aligned}
$$

where

$$
\rho^{*}=\frac{1}{2}{ }_{2} F_{1}\left(1,1, \frac{\alpha}{\lambda}+1, \frac{1}{2}\right) .
$$

Proof. The result follows by using the identity

$$
\begin{aligned}
\lambda z L_{a}^{t}(\alpha+1, \beta) f(z) & \\
= & {\left[(1-\lambda)\left(z L_{a}^{t}(\alpha, \beta) f(z)\right)+\lambda\left(z L_{a}^{t}(\alpha+1, \beta) f(z)\right)\right] } \\
& +(\lambda-1)\left(z L_{a}^{t}(\alpha, \beta) f(z)\right) .
\end{aligned}
$$

Remark 16. (i) We note that if $\lambda, \mu>0, \alpha=\beta=1$, and $t=0$ in Corollary 15, that is,

$$
\Re\left\{(1-\lambda)(z f(z))^{\lambda}+\lambda z^{2} f^{\prime}(z)(z f(z))^{\lambda-1}\right\}>\gamma,
$$

then (32) implies that

$$
\Re\{z f(z)\}^{\lambda}>\frac{2 \gamma+1}{2+1} \quad\left(z \in U^{*}\right)
$$

whereas if $f(z) \in \Sigma$ satisfies condition (61); then, by using Theorem 14, we have

$$
\mathfrak{R}\{z f(z)\}^{\lambda}>2(1-\ln 2) \gamma+(2 \ln 2-1)
$$

which is better than (61).

(ii) We observe that if $\lambda \in \mathbb{R}$ satisfying $\lambda \geq 0$ and

$$
k(z)=\frac{L_{a}^{t}(\alpha+1, \beta) f(z)}{L_{a}^{t}(\alpha+1, \beta) g(z)}+\left(\frac{1}{\lambda}-1\right) \frac{L_{a}^{t}(\alpha, \beta) f(z)}{L_{a}^{t}(\alpha, \beta) g(z)} .
$$

Then, from Theorem 10 (for $\mu=1$ ), we have that

$$
\mathfrak{R}\{k(z)\}>\frac{\gamma}{\lambda}
$$

implies

$$
\mathfrak{R}\left\{\frac{L_{a}^{t}(\alpha, \beta) f(z)}{L_{a}^{t}(\alpha, \beta) g(z)}\right\}>\frac{2 \alpha \gamma+\lambda \delta}{2 \alpha+\lambda \delta}
$$

whenever

$$
\mathfrak{R}\left\{\frac{L_{a}^{t}(\alpha, \beta) g(z)}{L_{a}^{t}(\alpha+1, \beta) g(z)}\right\}>\delta, \quad 0 \leq \delta<1 .
$$

Let $\lambda \rightarrow+\infty$; then, from (66), we have that $\mathfrak{R}\{k(z)\}>0$ implies

$$
\mathfrak{R}\left\{\frac{L_{a}^{t}(\alpha, \beta) f(z)}{L_{a}^{t}(\alpha+1, \beta) g(z)}\right\}>1, \quad z \in U^{*},
$$

whenever

$$
\mathfrak{R}\left\{\frac{L_{a}^{t}(\alpha, \beta) g(z)}{L_{a}^{t}(\alpha+1, \beta) g(z)}\right\}>\delta \quad\left(0 \leq \delta<1, z \in U^{*}\right) .
$$

In the following theorem, we will extend the above results as follows.

Theorem 17. Suppose that the functions $f(z)$ and $g(z)$ are in $\Sigma$, and suppose that $g(z)$ satisfies condition (14). If

$$
\mathfrak{R}\left\{\frac{L_{a}^{t}(\alpha+1, \beta) f(z)}{L_{a}^{t}(\alpha+1, \beta) g(z)}-\frac{L_{a}^{t}(\alpha, \beta) f(z)}{L_{a}^{t}(\alpha, \beta) g(z)}\right\}>\frac{(1-\gamma) \delta}{2 \alpha}
$$

$\left(0 \leq \gamma<1, \alpha \in \mathbb{R} \backslash\{0\}, 0 \leq \delta<1, z \in U^{*}\right)$, then

$$
\mathfrak{R}\left\{\frac{L_{a}^{t}(\alpha, \beta) f(z)}{L_{a}^{t}(\alpha+1, \beta) g(z)}\right\}>\gamma,
$$

$$
\Re\left\{\frac{L_{a}^{t}(\alpha+1, \beta) f(z)}{L_{a}^{t}(\alpha+1, \beta) g(z)}\right\}>\frac{(2 \alpha+1+\delta) \gamma-\delta}{2 \alpha} .
$$


Proof. Let

$$
q(z)=\frac{1}{(1-\gamma)}\left\{\frac{L_{a}^{t}(\alpha, \beta) f(z)}{L_{a}^{t}(\alpha, \beta) g(z)}-\gamma\right\} .
$$

Then $q(z)$ is analytic in $U^{*}$ with $q(0)=1$. Putting

$$
\Phi(z)=\frac{L_{a}^{t}(\alpha, \beta) g(z)}{L_{a}^{t}(\alpha+1, \beta) g(z)},
$$

we observe that by hypothesis $\Re\{\Phi(z)\}>\delta$, in $U^{*}$. A simple computation shows that

$$
\begin{aligned}
& \frac{(1-\gamma) z q^{\prime}(z) \Re\{\Phi\}}{\alpha} \\
& =\frac{L_{a}^{t}(\alpha+1, \beta) f(z)}{L_{a}^{t}(\alpha+1, \beta) g(z)}-\frac{L_{a}^{t}(\alpha, \beta) f(z)}{L_{a}^{t}(\alpha, \beta) g(z)} \\
& =\Psi\left(q(z), z q^{\prime}(z)\right),
\end{aligned}
$$

where

$$
\Psi(r, s)=\frac{(1-\gamma) \Phi(z) s}{\alpha}, \quad \alpha \in \mathbb{R} \backslash\{0\} .
$$

Using the hypothesis (70), we obtain

$$
\begin{aligned}
& \Psi\left(q(z), z q^{\prime}(z) ; z \in U^{*}\right) \subset \Omega \\
& \quad=\left\{w \in \mathbb{C}: \mathfrak{R}(w)>-\frac{\delta(1-\gamma)}{2 \alpha}\right\} .
\end{aligned}
$$

Now, for all real $r_{2}, s_{1} \leq-\left(\left(1+r_{2}^{2}\right) / 2\right)$, we have

$$
\begin{aligned}
\Re\left\{\Psi\left(i r_{2}, s_{1}\right)\right\} & =\frac{s_{1}(1-\gamma) \Re\{\Phi\}}{\alpha} \\
& \leq-\frac{\delta(1-\gamma)\left(1-r_{2}^{2}\right)}{2 \alpha} \\
& \leq-\frac{\delta(1-\gamma)}{2 \alpha} .
\end{aligned}
$$

This shows that $\mathfrak{R}\left\{\Psi\left(\mathrm{ir}_{2}, s_{1}\right)\right\} \notin \Omega$ for each $z \in U^{*}$.

Hence by Lemma 7, we have that this proves (71). The proof of (72) follows by using (71) and (72) in the identity:

$$
\begin{aligned}
\mathfrak{R}\left\{\frac{L_{a}^{t}(\alpha+1, \beta) f(z)}{L_{a}^{t}(\alpha+1, \beta) g(z)}\right\} & \\
= & \left\{\frac{L_{a}^{t}(\alpha+1, \beta) f(z)}{L_{a}^{t}(\alpha+1, \beta) g(z)}-\frac{L_{a}^{t}(\alpha, \beta) f(z)}{L_{a}^{t}(\alpha, \beta) g(z)}\right\} \\
& +\frac{L_{a}^{t}(\alpha, \beta) f(z)}{L_{a}^{t}(\alpha, \beta) g(z)} .
\end{aligned}
$$

This completes the proof of Theorem 17.
Remark 18. (i) Putting $\alpha=\beta=1, t=0$, and $g(z)=1 / z$ in Theorem 17 for all $z \in U^{*}$, we obtain that

$$
\mathfrak{R}\left\{z f(z)+z^{2} f^{\prime}(z)\right\}>-\frac{\delta(1-\gamma)}{2}
$$

implies $\mathfrak{R}\{z f(z)\}>\gamma$ and

$$
\mathfrak{R}\left\{2 z f(z)+z^{2} f^{\prime}(z)\right\}>\frac{(2+\delta) \gamma-\delta}{2} .
$$

(ii) For $\alpha=2, \beta=1, t=0$, and $g(z)=1 / z$ in Theorem 17 for all $z \in U^{*}$, we have that

$$
\Re\left\{z L_{a}^{t}(3,1) f(z)-z L_{a}^{t}(2,1) f(z)\right\}>-\frac{(1-\gamma) \delta}{4}
$$

$(0 \leq \gamma<1,0 \leq \delta<1)$ implies

$$
\begin{gathered}
\Re\left\{z L_{a}^{t}(2,1) f(z)\right\}>\gamma, \\
\Re\left\{z L_{a}^{t}(3,1) f(z)\right\}>\frac{(4+\delta) \gamma-\delta}{4} .
\end{gathered}
$$

\section{References}

[1] S. G. Krantz, "Meromorphic functions and singularities at infinity," in Handbook of Complex Variables, pp. 63-68, Birkhuser, Boston, Mass, USA, 1999.

[2] K. Knopp, "Meromorphic functions," in Theory of Functions Parts I and II, Two Volumes Bound as One, Part II, chapter 2, pp. 34-57, Dover, New York, NY, USA, 1996.

[3] H. M. Srivastava and A. A. Attiya, "An integral operator associated with the Hurwitz-Lerch zeta function and differential subordination," Integral Transforms and Special Functions, vol. 18, no. 3-4, pp. 207-216, 2007.

[4] H. M. Srivastava and J. Choi, Series Associated with the Zeta and Related Functions, Kluwer Academic Publishers, Dordrecht, The Netherlands, 2001.

[5] H. M. Srivastava, D. Jankov, T. K. Pogány, and R. K. Saxena, "Two-sided inequalities for the extended Hurwitz-Lerch zeta function," Computers \& Mathematics with Applications, vol. 62, no. 1, pp. 516-522, 2011.

[6] H. M. Srivastava, R. K. Saxena, T. K. Pogány, and R. Saxena, "Integral and computational representations of the extended Hurwitz-Lerch zeta function," Integral Transforms and Special Functions, vol. 22, no. 7, pp. 487-506, 2011.

[7] J. Dziok and H. M. Srivastava, "Certain subclasses of analytic functions associated with the generalized hypergeometric function," Integral Transforms and Special Functions, vol. 14, no. 1, pp. 7-18, 2003.

[8] F. Ghanim and M. Darus, "A new class of meromorphically analytic functions with applications to the generalized hypergeometric functions," Abstract and Applied Analysis, vol. 2011, Article ID 159405, 10 pages, 2011.

[9] F. Ghanim and M. Darus, "Some properties of certain subclass of meromorphically multivalent functions defined by linear operator," Journal of Mathematics and Statistics, vol. 6, no. 1, pp. 34-41, 2010.

[10] F. Ghanim and M. Darus, "New subclass of multivalent hypergeometric meromorphic functions," International Journal of Pure and Applied Mathematics, vol. 61, no. 3, pp. 269-280, 2010. 
[11] J.-L. Liu and H. M. Srivastava, "Certain properties of the DziokSrivastava operator," Applied Mathematics and Computation, vol. 159, no. 2, pp. 485-493, 2004.

[12] J.-L. Liu and H. M. Srivastava, "Classes of meromorphically multivalent functions associated with the generalized hypergeometric function," Mathematical and Computer Modelling, vol. 39, no. 1, pp. 21-34, 2004.

[13] S. S. Miller and P. T. Mocanu, Differential Subordinations: Theory and Applications, vol. 225 of Monographs and Textbooks in Pure and Applied Mathematics, Marcel Dekker, New York, NY, USA, 2000.

[14] S. Ponnusamy, "Differential subordination and Bazilevič functions," Proceedings of the Indian Academy of Sciences. Mathematical Sciences, vol. 105, no. 2, pp. 169-186, 1995.

[15] E. T. Whittaker and G. N. Watson, A Course of Modern Analysis: An Introduction to the General Theory of Infinite Processes and of Analytic Functions; With an Account of the Principal Transcendental Functions, Cambridge Mathematical Library, Cambridge University Press, Cambridge, Mass, USA, 4th edition, 1996. 


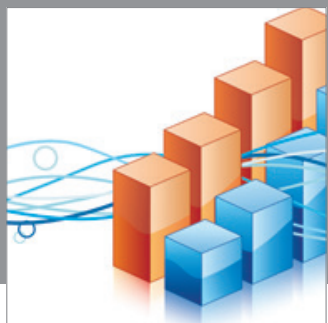

Advances in

Operations Research

mansans

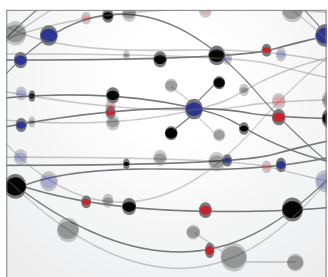

The Scientific World Journal
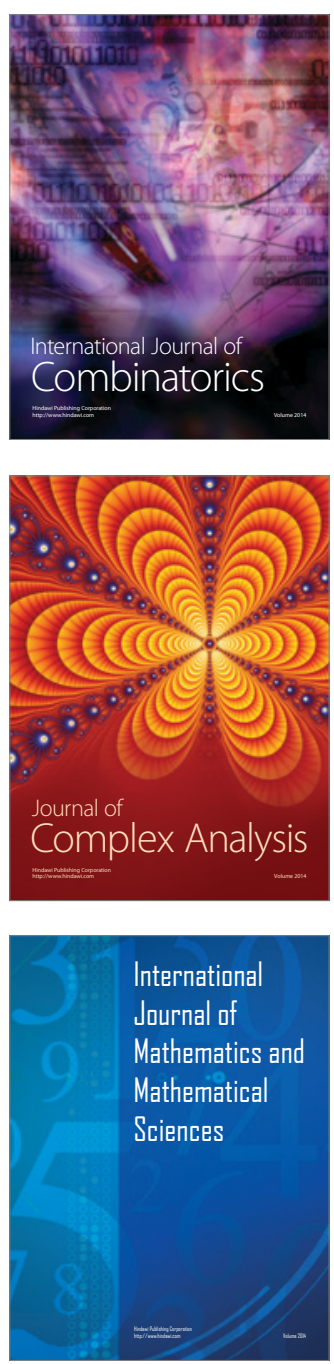
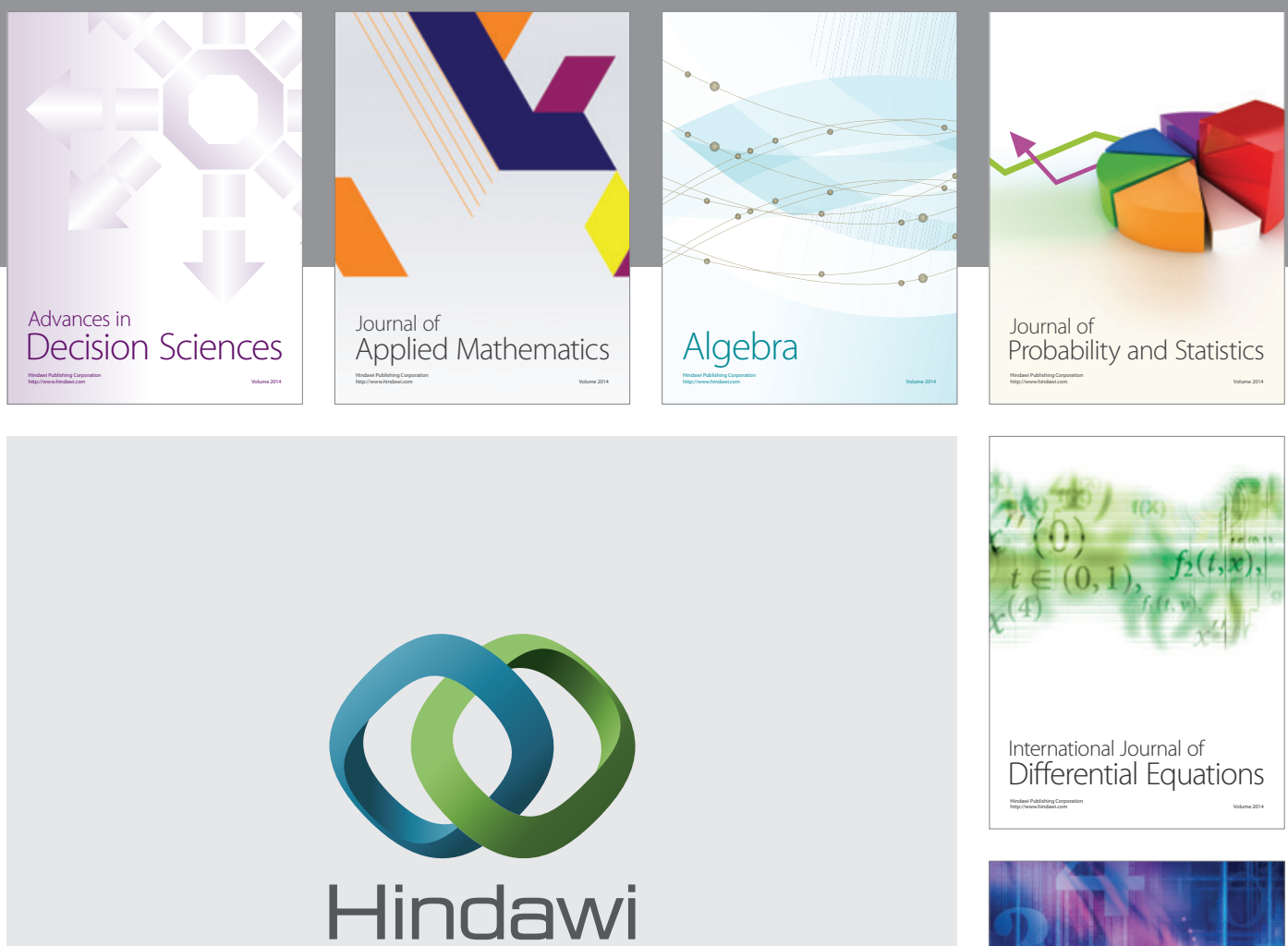

Submit your manuscripts at http://www.hindawi.com
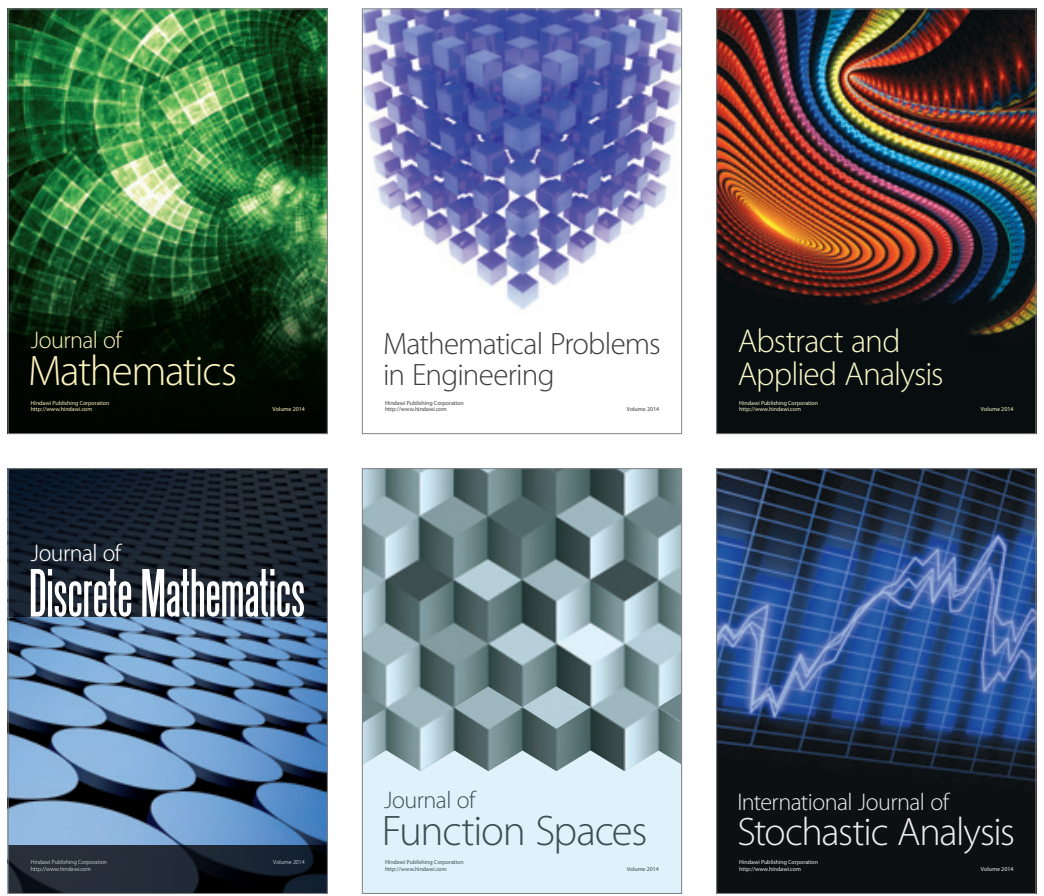

Journal of

Function Spaces

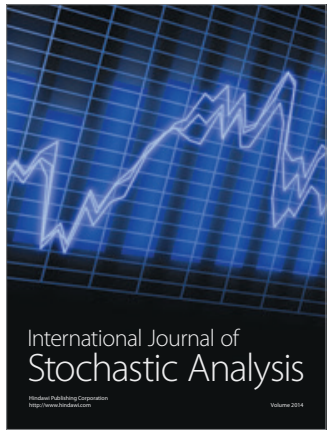

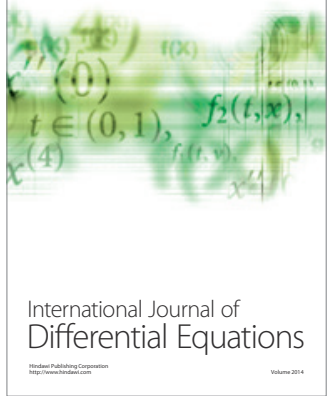
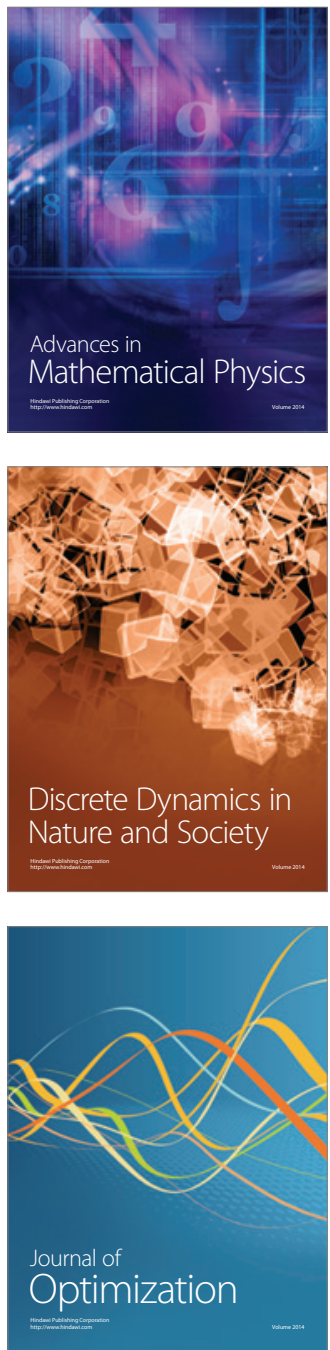